\title{
TESTIGOS DE JEHOVÁ E IMAGINARIO NORMALISTA EN LA ESCUELA PRIMARIA: IDENTIDADES EN COLISIÓN*
}

\author{
Por \\ José Luis Molina Hernández**
}

\begin{abstract}
RESUMEN
Problema llamativo y a la vez poco abordado, el conflicto que enfrenta a los testigos de Jehová y los profesores de educación básica en el marco de las escuelas públicas tiene implicaciones que trascienden a los actores. En este artículo nos centramos fundamentalmente en el caso de los profesores, como la parte menos estudiada del conflicto (lo que no significa reducir el problema o achacárselo a ellos), y desde los aportes de las teorías de la identidad, del análisis histórico gremial, y de la revisión de las implicaciones fenomenológicas de su vida cotidiana, tratamos de entender su posición y sus repuestas. La indagación sugiere que buena parte de sus posiciones con respecto a los símbolos patrios están determinados por factores de orden político-histórico, fenomenológicos, psicológicos, religiosos y ético-laborales, aunque tengan consecuencias pedagógicas no intencionadas.
\end{abstract}

\begin{abstract}
Eyecatching problem and at the same time mostly overlooked, the conflict among the Jehova's Witnesses and the elementary school teachers in public schools has implications that transcend to the actors. In this article we are focused on the teachers, as the less studied part of the conflict (this does not mean to minimize the problem, or make them responsible), and from the contributions of identity theories, the gremial historic analysis, and from the revision of the phenomenological implications of their daily life, we try to understand their posture and their answers. The indagation suggests that an important part of their postures respect to patiotic symbols is determined by political-historical factors, phenomenological factors, and psychological, religious, and ethnical-labor factors, even though they have no intended pedagogical consequences.
\end{abstract}

* Ensayo presentado en el colEr-Estudiantil II, en El Colegio de la Frontera Norte, en Tijuana, B.C., en enero de 1996. Se agradecen las observaciones, comentarios y críticas de Gilberto Giménez (IIS-UNAM), Jorge Durand (U. de G.), Jorge Alonso (CIESAs Occidente), Jorge Bustamante, José Manuel Valenzuela y Claudia Campillo (COLEF).

** Estudiante del Doctorado en Ciencias Sociales de El Colegio de la Frontera Norte, y profesor-investigador de la Universidad Pedagógica Nacional-Unidad Mexicali.

N. del E. Este artículo se aprobó en mayo de 1977 para publicarse en Estudios Fronterizos. 


\section{INTRODUCCIÓN}

Para el lector habitual de periódicos, una nota recurrente es la de que se expulsó a niños testigos de Jehová de una escuela primaria por no hacer honores a la bandera; esto lo mismo en el sureste del país, que en la frontera norte o en la región central. La recurrencia y ubicuidad de esta contradicción la ubican como un problema social y sociorreligioso actual y apremiante, sobre todo en la medida en que los más afectados, los nif́os, son precisamente los menos responsables de las preferencias simbólicas y las definiciones ideológicas de sus mayores. En este sentido, se justifica el esfuerzo analítico que se haga en este campo.

Cuando se aborda esta cuestión la atención se centra casi siempre en los testigos de Jehová, como el elemento problemático, donde éstos aparecen como retadores y aun subversivos respecto a los valores cívicos hegemónicos. Sin embargo, se hace abstracción del papel de otros agentes participantes en el conflicto, en particular el de los profesores de educación básica. ${ }^{1}$ En este trabajo nos proponemos, precisamente, someter a examen esa parte inexplorada, mediante el análisis de algunos de los rasgos identitarios de los docentes y cómo es que entran en contradicción con la fuerte identidad desarrollada por los testigos de Jehová. Para ello nos apoyaremos tanto en algunos conceptos sociológicos como en las nociones de instituciones voraces, circuitos de pertenencia, e identidades crispadas, entre otros, así como en informaciones recogidas en entrevistas ex profeso y en el conocimiento previo de los campos en estudio. ${ }^{2}$

\section{EL PROBLEMA DE LAS ADSCRIPCIONES IDENTITARIAS}

Por principio de cuentas, habría que considerar que tanto la nación como los grandes movimientos y grupos religiosos son comunidades imaginadas, ya que los miembros de éstas comparten un rasgo identitario central, un símbolo o conjunto de símbolos que los unen, al margen de que jamás en su vida tengan contacto directo o sepan siquiera de su existencia; basta, afirma Anderson, con que en la mente de cada uno de ellos permanezca la imagen de su comunión, experimentada como una camaradería profunda y horizontal. (Anderson, 1983:15-16).

En el caso de las comunidades representadas por los testigos de Jehová y los símbolos patrios, se trata, hasta cierto punto, de instituciones voraces

1 Un ejemplo de este sesgo puede verse en Canto Ramírez, 1991.

2 He venido estudiando fenómenos del campo religioso desde 1988; a este tema dediqué mis tesis de licenciatura y maestría. 
(Cosler, 1978:11-17, 101-112), es decir, aquéllas que tienden a controlar todas las adscripciones del sujeto exigiéndole lealtad a un jefe, un grupo o una idea. Decimos hasta cierto punto porque esta idea debe ser matizada desde dos ángulos distintos: en el caso del nacionalismo, no hay que perder de vista que éste contiene un componente universalista (o coexiste con él), que es lo que permite que otros códigos simbólicos estén presentes en una misma identidad (por ejemplo: ser mexicano, por un lado, católico, apostólico y romano, por otro, y miembro de la Asociación latinoamericana de sociología); así, el nacionalismo puede coexistir tanto con la afiliación partidaria, como con la mayor parte de las identidades religiosas, y con la asimilación de pautas de consumo y de comportamiento provenientes del exterior del país.

El grupo religioso en cuestión, es sin duda uno de los más absorbentes - es una religión de exigencia, dice orgulloso un testigo de Jehová-, y tiende a poner todas las prácticas sociales bajo la observancia de sus normas religiosas particulares; no obstante, el grado de absorción no alcanza los niveles que se registran en grupos como Hare Krishna o los Niños de Dios (Woodrow, 1979), por citar sólo dos ejemplos, que segregan al individuo de la estructura familiar, laboral, escolar, etcétera, esto es, que se apropian totalmente de la vida social de sus adherentes. Por vía distinta, y con todas las diferencias del caso, esto se aplica también a los mennonitas y de otras sectas introversionistas. (Wilson, 1970).

Así pues, se trata de dos identidades densas, con tendencias excluyentes pero no exclusivas, y que por lo tanto no segregan al individuo del cuerpo social general, y toleran - en diversos grados- la presencia de otros rasgos identitarios en el sujeto. Dicho de otro modo, nacionalismo y afiliación a los testigos de Jehová suelen formar parte de circuitos de pertenencia ${ }^{3}$ más o menos plurales, y sólo entran en conflicto en determinadas circunstancias.

Veamos esto más en detalle: consideramos aquí que los circuitos de pertenencia, como las cadenas genéticas, varían de un individuo a otro lo mismo que de un grupo a otro. En los individuos las variaciones están influidas tanto por el componente biográfico como por el factor situacional o efecto contextual; lo que impide que las variaciones se den sin control hasta el infinito es la existencia de identidades colectivas concretadas en grupos - y cuyo fundamento sociohistórico y psicológico no podemos abordar en este ensayo - que tienden a homogeneizar no sólo las escalas

3 Concepto expuesto por Gilberto Giménez en la sesión del 27 de septiembre de 1995, en el Seminario Sociología de la cultura/Dinámica cultural socioterritorial, en el Programa de Doctorado en Ciencias Sociales de El Colegio de la Frontera Norte. 
de valores, sino también la configuración de los circuitos de pertenencia de sus miembros, estableciendo jerarquías entre dichas pertenencias. Esta posibilidad de normar las configuraciones reduce el número de identidades individuales diferenciadas. Por ello, se convierten en ejes de constitución de habitos semejantes, generando de esta manera, concepciones, prácticas y formas de respuesta social relativamente indiferenciadas para un mismo grupo.

La coexistencia de adscripciones distintas en los circuitos de pertenencia de un individuo son, luego entonces, un hecho normal, por lo cual no necesariamente chocan entre sí en tanto se remitan a campos distintos de la realidad, es decir, mientras en un caso se trate de lealtades políticas y en otro de afiliaciones religiosas, culturales o deportivas; pero aun en el caso de dos identidades religiosas diferentes - catolicismo y umbanda, y catolicismo y espiritismo, son ejemplos de coexistencia de adscripciones religiosas distintas en un mismo sujeto- no siempre llegan a excluirse como factores identitarios.

Lo que observamos en el caso que nos ocupa, en cambio, es un fenómeno de identidades crispadas, ${ }^{4}$ como si se tratara de dos organismos que reaccionaran biológicamente al mutuo contacto y se predispusieran al combate.

Si pensamos, por otro lado, que los testigos de Jehová no enfrentan mayores problemas en su papel de trabajadores, de vecinos o de consumidores, y que los símbolos patrios son respetados de manera más homogénea en la mayor parte de los ámbitos nacionales, podemos afirmar que se trata de una crispatura diferenciada, que varía su intensidad y sus manifestaciones según los actores, el tiempo y las circunstancias.

La pregunta es, entonces, por qué estas dos formas de identidad - testigos de Jehová y símbolos patrios- se crispan como lo hacen, en qué espacios, tiempos y circunstancias, y a través de qué actores concretos.

\section{LA RELIGIÓN DE LA PATRIA}

Si partimos de que la identidad es al mismo tiempo construcción mental y construcción objetual, tanto del observador, como señala Bourdieu (1982), como también de los propios actores, podemos establecer que el acendrado patriotismo de la sociedad mexicana es, si no más intenso, si por lo menos más exteriorizado y objetivado en los símbolos que el de otras sociedades, v.g. la norteamericana. En una sociedad como la nuestra el nacionalismo

\footnotetext{
4 Concepto expuesto por el mismo autor en la sesión citada en la nota anterior.
} 
deviene estatus adscrito por nacimiento, ya que tiene un carácter casi obligatorio desde el punto de vista moral. ${ }^{5}$

Por último, es importante recalcar la capacidad del nacionalismo de dar origen a una religión de la patria (Giménez, 1993:17), junto con una teología de la patria y su santoral y ritos respectivos.

\section{LA TRANSFORMACIÓN DEL CAMPO RELIGIOSO, MARCO INICIAL DE LA POLÉMICA}

En este contexto, analizaremos someramente uno de los polos de la contradicción, el de la polémica en el campo religioso.

El crecimiento de los grupos religiosos no católicos ${ }^{6}$ en una sociedad hasta hace poco tiempo formalmente monorreligiosa no deja de ser una fuente de tensión social; empero, un primer matiz a esta potencialidad de conflicto es la constatación de que el crecimiento de estos grupos se caracteriza por su heterogeneidad: las iglesias históricas (luteranos y calvinistas, por ejemplo) tienen un crecimiento casi vegetativo, en tanto que pentecostales y testigos de Jehová crecen a un ritmo acelerado. (Valderrey, 1988:29). Son precisamente estas dos corrientes religiosas, junto con los mormones, los que con mayor frecuencia, aunque por diferentes razones, entran en conflicto con el resto de la sociedad.

Por otra parte, el factor central de "otredad" de estos grupos se basa en su origen externo, ya que en su gran mayoría provienen de Estados Unidos y Europa. Este rasgo, sin embargo, se deriva de un corte temporal arbitrario, ya que el conjunto del cristianismo tienen un origen externo. Ciertamente la incorporación de algunos de los elementos de la simbólica católica al conjunto de símbolos patrios tiene un fundamento histórico, en la medida en que está presente en las grandes gestas populares - la independencia y la revolución, sobre todo- $\mathrm{y}$ en la vida cotidiana de los hogares mexicanos desde el siglo XVI, por lo cual se ha sedimentado junto con otros componentes no religiosos de la identidad nacional.

No obstante, cabe recordar que en los primeros años de vida de esta nación - y aun hasta el siglo pasado - los sectarios eran las hoy denominadas iglesias históricas, como el luteranismo, el calvinismo, el anglicanismo, etcétera, (Macín, 1983) a las que hoy se les reconoce cierta

5 No en vano el término "apátrida" tiene una connotación además de despectiva, de marginalidad, de hijo sin padre: el que no tiene patria no tiene identidad, es el "otro" siempre, atrapado, como el pachuco. Laberinto de la soledad ( $\mathrm{Paz}, 1975)$.

6 Dicho crecimiento ha sido abordado desde distintos ángulos por diversos investigadores como Bastián (1986), Valderrey (1988), Giménez (1988), Hernández (1989), Masferrer (1991), y Molina (1993), entre otros. 
respetabilidad por parte de la iglesia hegemónica; prueba de esto es que en tierras novohispanas el término "luterano" fue por más de trescientos años sinónimo de hereje. En nuestro siglo, los intrusos son los recién llegados $\mathrm{y}$, significativamente, los que mayor desafío implican dentro del campo religioso.

Ahora bien, de los grupos "incómodos", los de la corriente pentescostal - la Apostólica de la Fe, Asambleas de Dios y muchas más-mantienen mucho menos distancia con la corriente religiosa dominante y con el conjunto de la sociedad a nivel de símbolos religiosos y cívicos; sus principios doctrinales no contradicen la aceptación de los símbolos patrios, y por el contrario, suelen ser abiertamente nacionalistas. Los mormones, otra de esas identidades "non gratas", tienen por regla general un comportamiento escasamente contradictorio con las normas cívicas de los países en los que se implantan, por lo cual sus problemas en este sentido no existen; en su caso, las disputas tienen un fondo religioso más claro.

Con este proceso de destilación llegamos al grupo en el que las pasiones sociorreligiosas se enconan: los testigos de Jehová. Hasta aquí, todo parece confirmar que el problema básico reside en ellos, por lo cual es necesario detenerse un momento en sus rasgos característicos.

\section{TESTIGOS DE JEHOVÁ: RASGOS CONFLICTIVOS DE UNA IDENTIDAD HERMÉTICA}

No vamos a detenernos en la elaboración de una monografía de los testigos de Jehová, ya que esto ha sido realizado en diversos niveles y extensiones. Lo que nos interesa más bien es revelar algunas de las características del grupo y de los puntos de conflicto que se suscitan en torno suyo, y que lo hacen tan notorio socialmente hablando. Queremos mostrar, de esta manera, que la contradicción testigos de Jehová-símbolos patrios sólo adquiere relevancia en determinados contextos.

Los testigos de Jehová se caracterizan, entre otras cosas, por la consistencia de sus prácticas sociales, por su proselitismo activo, agresivo, constante, así como por la certeza que demuestran en sus convicciones religiosas. (Valderrey, 1988:31). Esta "densidad" práctico-simbólica que estabiliza los circuitos de pertenencia en una identidad social relativamente tensa y desafiante, es capaz incluso de funcionar como una patria sustitutiva, como en el caso de los polacos inmigrantes en el norte de Francia (Dericquebourg, 1977:71-82). Y aún más, los testigos se conciben como una nación religiosa (La Atalaya, 1 de enero de 1991:3-4), es decir, una

7 Por ejemplo Hebert (1960), Woodrow (1979), Giménez (1988). 
nación supranacional, que incluye la pertenencia a distintas identidades nacionales, sin necesariamente entrar en contradicción con ellas, aunque siempre estableciendo claramente sus límites ("nosotros no somos parte del mundo, como sí lo son los 'otros'", afirman sin titubear).

En realidad su conocida oposición a rendir honores a la bandera ${ }^{8}$ no es el único punto de fricción con su entorno, ya que para ellos existen diversas prohibiciones - a partir de un fundamento bíblico siempre- y no todas son estigmatizadas; ejemplo de ello es su oposición a ir a la guerra, así como su rechazo a participar en política, en concursos de belleza, a celebrar la navidad y los cumpleaños, entre otras, por no hablar de su rechazo al homosexualismo y a las relaciones premaritales. (Franz, 1989:9-10, 1625). En estos casos es raro que se produzca tensión en los medios en que se desenvuelven.

Una de sus restricciones que sí genera fuertes conflictos es la de las transfusiones de sangre. En otros contextos, como el de Estados Unidos?, esta es la principal contradicción con su entorno. Cuando esta negativa se ha relacionado con la vida de sus hijos o parientes, se les ha acusado de violadores de los derechos humanos. En todo caso, este problema ha tenido salida ya que, como afirma Selva Pallares (1992), los testigos no se oponen al tratamiento sino a la transfusión, y aceptan los sustitutos de sangre, la autotransfusión intraoperatoria y la hemodilución; también se apela en estos casos a las inyecciones de hierro dextran y otros estimuladores de glóbulos rojos como la eritropeyetina.

Se trata, pues, de un problema soluble con un poco de buena voluntad de las partes.

La contradicción entre testigos y símbolos patrios, por el contrario, está lejos de resolverse, y también a la inversa de aquél, los testigos aparecen aquí frecuentemente como víctimas de violación de los derechos humanos, tal como consta en las constantes denuncias ante las comisiones nacional y estatales de derechos humanos del país.

8 La Ley de Asociaciones religiosas y culto público, del 13 de julio de 1992, habla de la relación con los símbolos patrios sólo hasta su título quinto, relativo a las infracciones y sanciones; en el capítulo primero, artículo 29, fracción II, establece como infracción: "Agraviar a los símbolos patrios o de cualquier modo inducir a su rechazo" (Diario Oficial, 15 de julio de 1992:42). La discusión —a la cual los testigos dan un fundamento en parte religioso (v.g. Mateo 4:10; Éxodo 20:4,5) y en parte analítico (Williams, 1952; Manwaring, 1962) estriba en si negarse a saludar a la bandera constituye o no una forma de agravio o de inducir su rechazo.

9 Cabe aclarar que el problema del rechazo a saludar a la bandera ha tenido importancia en Estados Unidos en ciertas etapas de su historia, por ejemplo en los años treinta (Beckford, 1977:26). 
Notemos, pues, que el fenómeno en cuestión tiene lugar en un contexto bien determinado: a nivel macro, como ya se dijo, en una sociedad nacionalista, católica y monorreligiosa por cuatro siglos y medio a nivel particular, el problema se da preferentemente en el ámbito de la educación básica.

Es claro que la relación grupos religiosos-instituciones educativas no es contradictoria por sí misma (Bastián, 1989:143-171; Lamond Tullis, 1987:173-200) ¿qué es lo que hace entonces que esta multicitada fórmula resulte potencialmente conflictiva? ¿por qué específicamente en la educación básica y con los testigos de Jehová?

\section{LOS GUARDIANES DE LA PATRIA}

Es hora de realizar un acercamiento a algunas de las características de la identidad del docente que permiten entender el porqué de este enfrentamiento radical. Además del nacionalismo retórico de los políticos, hay dos sectores que se conciben a sí mismos como los defensores de la nación, la nacionalidad y el nacionalismo. El primero de ellos es el ejército, el cual, por su propia naturaleza, no acostumbra discutir en absoluto la legitimidad de esta identidad casi obligatoria. La verticalidad total y la fuerza de las armas son para ellos razones suficientes para resguardar la integridad de la patria.

El otro espacio de atrincheramiento de la concepción hegemónica de la identidad nacional, o, como dice Giménez siguiendo por un lado a Castoriadis y por otro a Benedict Anderson, de lo imaginario nacional (Giménez, 1993:15) es el espacio de la educación básica, es decir, los niveles de preescolar, primaria y secundaria, que es donde se acostumbra celebrar honores a la bandera. Aquí el asunto cobra relevancia porque se inserta en el debate de los símbolos; si con el ejército no existe posibilidad de discusión, con el magisterio, que es representante de una parte del conocimiento formal socialmente legítimo, la polémica resulta del todo natural.

\section{FACTORES CONSTITUTIVOS DE LA IDENTIDAD SOCIAL DEL DOCENTE}

Es necesario tratar de reconstruir, así sea someramente, algunos de los rasgos característicos de la identidad del docente, que nos permitan entender su posición respecto a los símbolos patrios; veremos, de paso, como este énfasis en dichos símbolos no es constante a lo largo de su historia, aún cuando permanezca como parte de su discurso. 
Entre los múltiples rasgos que la indagatoria de Bonifacio Barba y Margarita Zorrilla destaca como característicos del docente de educación básica, está el de concebir al nacionalismo como un interés común de todos los mexicanos (Barba y Zorrilla, 1987). En esta concepción de sí mismos convergen razones históricas, fenomenológicas, psicológicas, y de otro tipo; a continuación revisaremos brevemente cada una de ellas.

1) Dimensión política-histórica: La etapas que distingue Reyes Esparza (1988), pese a su carácter elemental, permiten una aproximación útil para nuestros propósitos. De acuerdo con él, inicialmente el sacerdote es el que hacía las veces de profesor, tanto en la época prehispánica como en los primeros años de la Colonia; en la etapa siguiente, que cubre el resto de la época colonial hasta los inicios del México independiente, surge la docencia como una actividad regulada, pero con la característica de carecer de una preparación profesional.

Es hasta la tercera etapa cuando se inicia el esfuerzo para preparar a los docentes a través de las normales lancasterianas; aunque su repercusión es mínima, lo relevante es que en tanto esfuerzo de educación masiva están articuladas con el proyecto liberal de sociedad y su confrontación con la Iglesia Católica: el proyecto político de los liberales suponía la creación de una sociedad libre, "colocada por encima de los privilegios del clero y la milicia... [y] no es posible pensar en la formación de una sociedad de hombres libres sin un proceso educativo previo" (Álvarez y Limón, 1984:54); el surgimiento del gremio, entonces, aparece marcado por esta confrontación, lo que genera un profesor liberal, articulado al Estado y, formalmente, al menos, antieclesial. En este sentido, el magisterio deviene factor esencial en la conformación de una idea particular de la identidad nacional.

En la etapa siguiente, con el desarrollo de las normales inspiradas -entre otras fuentes- en las ideas de Rébsamen (1891), y en la visión positivista comtiana introducida por Gabino Barreda entre 1863 y 1877 (Barreda, 1987), el profesor se caracteriza por su defensa del orden establecido como vía para alcanzar el progreso; esta ideología no lo convierte en antirreligioso pero sí lo lleva a menospreciar el aporte de las experiencias "no científicas" en el ámbito educativo, y por lo tanto a mantener una actitud distante respecto a la religión (acorde con la relativa tolerancia porfirista hacia la Iglesia Católica).

La quinta etapa es posterior al movimiento revolucionario y está marcada por uno de los símbolos más fuertes en la identidad docente actual: el maestro rural. Se ha escrito abundantemente sobre este prototipo como para hacer necesaria una mayor exposición; sólo queremos aquí destacar los siguientes rasgos: tanto su activismo social que trascendió con 
mucho su labor en el aula, como la presencia de militantes comunistas que culmina con el proyecto -inconcluso- de educación socialista, marcan el carácter francamente antirreligioso de esta etapa. Una paradoja adicional es que este símbolo tan fuerte para el normalismo contemporáneo fue generado por profesores que en su gran mayoría no fueron normalistas. (Zúniga 1993a:19).

Finalmente, en el reflujo del cardenismo y su desmantelamiento parcial, la idea de la unidad nacional promovida por Ávila Camacho y sus sucesores centró su base en la educación básica, y en particular en la escuela primaria; si bien el tema de la patria ha sido siempre un factor fuerte para el magisterio desde su surgimiento, ahora se convierte en un eje axial, en centro explícito a partir del cual se dan los procesos formativos. El niño se forma, ante todo, como un futuro ciudadano.

Como el mismo Reyes Esparza reconoce, esta etapa permite un mayor grado de especificidad, por lo que habría que ver qué rasgos particulares incorpora a la identidad del docente la reforma educativa de 1970, por un lado, y el proyecto actual de modernización educativa con su propuesta de descentralización y regionalización.

En todo caso, es posible plantear, a nivel de hipótesis, que estos rasgos históricos, junto con otros de naturaleza diversa que confluyen en la vida cotidiana del docente, se condensan dando lugar a ese complejo simbólico que denominamos la identidad del docente.

2) Dimensión fenomenológica: La aproximación a los espacios y el actuar cotidiano de los docentes es posible tomando como base la noción de Shutz de mundo de vida. (Shutz, 1974).

Dado el lugar principal que ocupa en su práctica cotidiana -el que lo define en última instancia- que es frente a y al frente de un grupo de alumnos con un desarrollo físico e intelectual menor que el suyo, el docente experimenta en sus actividades cotidianas el poder de definir espacios (con las restricciones que imponen los flujos dialécticos de poder que se registran siempre entre los grupos, los cuales impiden que el poder se concentre de manera absoluta en un solo polo). Hay, en este sentido, una sensación de omnipotencia. (Neill, 1975).

De acuerdo con esto, si cada gremio reivindica en alguna forma su poder sobre su espacio de trabajo, el docente tiene claro, o cree tenerlo, que las definiciones fundamentales de la vida escolar le corresponden, y en particular las que tienen que ver con la formación de los sujetos y el desarrollo de la sociedad: "se considera como vanguardia de una nueva utopía y percibe su acción como un apostolado moderno"(Barba y Zorrilla, 1987:32); y esto, con el tono sumamente afectivo, emocional, con que carga todas las representaciones que se refieren a él y a su quehacer, y que 
lo llevan a concebirse como el responsable, en buena medida, del destino de la humanidad: "forjador del futuro, formador de hombres, sembrador de simientes de esperanza, constructor de la patria, etcétera." (Zúniga, 1993b:43).

3) Dimensión psicológica. Considerado como sujeto psíquico, la posición fenomenológica del docente puede verse como anudada en los factores irracionales de la subjetividad, y expresada en "las representaciones imaginarias, fantasías y deseos que la sociedad, los estudiantes y los propios maestros normalistas, tienen y proyectan sobre la profesión docente." (Zúñiga, 1993b:42). De esta manera se conforma una identidad paradigmática de importante presencia en México: el normalismo, y, correlativamente, el imaginario normalista, ${ }^{10}$ es decir, el conjunto de símbolos, emblemas identificatorios y prácticas sociales, que enmarcan y guían la rutina laboral del docente.

Pero éste, afirma Zúñiga, deviene un imaginario alienante, ya que "el normalista es para sí mismo tal como lo describen sus metáforas identificatorias: el sembrador de semillas que germinarán" (Zúniga, 1993a:21), de ahí que el currículo real en la formación de maestros y en la educación básica en general no esté centrado en el conocimiento sino en el cumplimiento de las normas moralizantes, de los principios éticos que el docente se siente impelido a trasmitir y defender.

Tenemos, pues, un docente que es a la vez dominante y dominado, idealizado y subestimado, mitificado-mistificado (mediante la exaltación de la vocación que, de paso, afecta negativamente la profesionalización) y rutinizado:

Mirando a un pasado mítico se escapan de [su práctica docente] aspirando a dejar el magisterio, a veces incluso, asumen el menor compromiso posible, porque saben que a pesar de las difíciles e innumerables tareas y dificultades que enfrentan diariamente y del esfuerzo que hacen por sacar adelante su trabajo profesional, seguirán siendo menospreciados por la sociedad y por el resto de los trabajadores intelectuales y profesionales... (Zúñiga, 1993a:20-21).

4) Dimensión religiosa: Hay una contradicción religiosa subyacente que no puede soslayarse en un contexto como el de México, un país que hasta hace poco era casi totalmente monorreligioso, y que lo es aún en

\footnotetext{
${ }^{10}$ No está por demás notar que tanto la noción de imaginario normalista, de Zuñiga, como la de imaginario nacional, de Giménez, parten del concepto de imaginario social, de Castoriadis, y en nuestra perspectiva ambos confluyen de manera fundamental en la identidad del docente.
} 
muchas regiones (Aguascalientes, por ejemplo). En un medio religioso altamente uniforme como este, no es raro que las identidades religiosas entren en conflicto. Cabe pensar, entonces, que así como el conjunto de la población es definido casi en su totalidad por su adscripción religiosa, y mayoritariamente católica, el gremio de los maestros debe tener una definición semejante, y que por tanto la mayor parte de los maestros profesan alguna religión y que los más son católicos.

Esto plantea una paradoja interesante: el docente en tanto gremio específico nace por oposición al dominio religioso en la educación, desde el positivismo desdeña las creencias metafísicas, como activista rural llega hasta combatir a la religión, y en el esquema de la unidad nacional le delimita celosamente sus espacios, pero, no obstante ello, la mayor parte del magisterio mantiene sus creencias religiosas católicas.

Lo importante para nuestra discusión, en todo caso, es que aun cuando se trate de católicos pasivos o nominales, resulta lógico que frente a una identidad religiosa fuerte y perturbadora como la de los testigos, se produzca una reacción de la adscripción religiosa subyacente; cabe suponer, pues, que en una situación de tensión identitaria, simbólica, la identidad religiosa de origen se manifiesta también en la confrontación con el grupo mencionado. (En el caso de los no creyentes, que también representan una cuota importante dentro del magisterio, la contradicción ideológica es más amplia, ya que cuestiona la existencia misma de lo religioso e incluye, por tanto, la discusión con el catolicismo).

5) Dimensión ético-laboral: Con base en observaciones preliminares y de sentido común, es posible aventurar también que hay una contradicción entre concepciones del trabajo, éticas laborales y prácticas de trabajo cotidianas. Los testigos son considerados generalmente por sus propios maestros como alumnos más disciplinados, trabajadores y ordenados que la mayoría de sus compañeros. Los docentes, en cambio, mantienen una relación ambigua con lo laboral que oscila entre la idealización emotiva y el abandono en la práctica, como hemos dicho antes; ejemplos de ello son la lucha contra el calendario de los 200 días, las constantes suspensiones de clases, y la constancia de los poros del trabajo, hechos discutidos constantemente por los padres de familia, las autoridades, y desde luego, por los propios profesores.

A nivel meramente conjetural, podemos decir que esta diferencia también puede reforzar la sensación de alteridad e introducir elementos de tensión inconsciente. 


\section{CONCLUSIÓN: CONFLICTO SIMBOLICO EN EL ESPACIO "SAGRADO" DE LA ESCUELA}

Lo que analizamos a lo largo de este trabajo, son los factores por los cuales entran en conflicto los circuitos de identidad patria e identidad religiosa en colectivos con diferente jerarquización de los valores que configuran tales circuitos. El imaginario normalista, base de la identidad del maestro de educación básica, firme, homogénea hacia el exterior (no tanto al interior), y alimentada por mitos que tienen un sustrato a la vez social y religioso, frente a otra, la de los testigos de Jehová, también firme, radical, aun más homogénea - prácticamente monolítica-, e igualmente fundada en mitos irreductibles.

Pero esto se da casi exclusivamente en un espacio, el de la educación básica, ya que si los mismos actores se encuentran en otros espacios - en los que los símbolos no están en cuestión-como un parque o un centro comercial, no se genera ningún enfrentamiento. Si esto es así es porque la escuela es la iglesia de la religión de la patria (y desde el momento en que la religión católica se reclama como parte esencial de la identidad nacional, se convierte en parte de la religión de la patria), con sus símbolos no racionalizados ni homogeneizados, y porque en ella los oficiantes son los docentes, que así alimentan sus propios mitos.

Lo que importa no es la sacralidad de los emblemas, sino la legitimación de los actores y la delimitación del dominio simbólico sobre el espacio social; por lo tanto, lo que entra en conflicto con los testigos de Jehová no son los símbolos patrios sino el imaginario normalista.

Concluimos, pues, que hay un fondo pararreligioso en esta disputa, sólo que en uno de los actores se expresa en términos cívicos, mientras que en el otro la respuesta se da a través de un discurso casi totalmente religioso. Cada quien tiene sus propios espacios sagrados y profanos, sus narrativas de salvación, sus héroes, símbolos y estrategias de práctica social. Pero al confluir en el tiempo y en el territorio los mitos fundantes se vuelven repentinamente contrarios, se repelen, y, si predominan los instintos sobre la razón, sobreviene la confrontación.

Tenemos, en resumidas cuentas, dos identidades en colisión, y en medio de ellos, un símbolo. 


\section{BIBLIOGRAFIA}

ÁLVAREZ Barret, Luis, y Miguel Limón R. 1984. "El artículo 30. constitucional", en: Antología de política educativa en México II, UPN, México.

ANDERSON, Benedict. 1983. Imagined Communities: Reflection on the Origin and Spread of Nationalism. Londres, Verso Editions.

BARBA, José B., y Margarita Zorrilla. 1987. "Un trabajador llamado maestro", en: Pedagogía, vol. 6, núm. 19, julio-septiembre de 1989, México.

BARREDA, Gabino. 1987. La educación positivista en México. Editorial Porrúa, México.

BASTIÁN, Jean Pierre. 1983. Protestantismo y sociedad en México.Casa Unida de Publicaciones, México.

- 1986. Historia del protestantismo en América Latina. CUPSA, México.

- 1989. Los disidentes. Sociedades protestantes y revolución en México, 1872-1911. FCE.-El Colegio de México, México.

BECKFORD. 1977. “Jehovah's Witnesses World-Wide", en: Social Compass, vol. XXIV, 1977/1.

BOURDIEU, Pierre. 1982. "La identidad como representación", en: $\mathrm{La}$ teoría y el análisis de la cultura (Gilberto Giménez, coordinador). SEP-Universidad de Guadalajara-COMECSO.

CANTO Ramírez, José Luis. 1991. Las sectas religiosas y su influencia en la deserción escolar en las zonas rurales del estado de Campeche. Documento preliminar, UPN-Unidad 041, Campeche.

COSLER, Lewis A. 1978. Las instituciones voraces. FCE, México.

DERICQUEBOURG, Regis. 1977. "Les Temoins de Jéhovah dans le Nord de la France: Implantation et Expansion", en: Social Compass, vol. XXIV, 1977/1.

FRANZ, F.W. 1989. La escuela y los testigos de Jehová. Watch Tower Bible and Tract Society of Pennsylvania. Grupo Editorial Ultramar, México.

GIMÉNEZ, Gilberto. 1982. Para una concepción semiótica de cultura. Material mimeografiado. México.

- 1988. Sectas religiosas en el sureste: aspectos sociográficos y estadísticos. Centro de Investigaciones y Estudios Superiores de Antropología Social, CIESAS del Sureste, Cuadernos de la Casa Chata 161, México, D.F. 
- 1989. "Nuevas dimensiones de la cultura popular: las sectas religiosas en México", en: Estudios sobre las culturas contemporáneas, vol. III, núm. 7, Colima.

- 1993. "Apuntes para una teoría de la identidad nacional", en: Sociológica, año 8, número 21. Identidad nacional y nacionalismos, enero-abril de 1993, México.

HEBERT, Gérard. 1960. Les Temoins de Jéhovah. Essai Critique d' histoire et de Doctrine. Les Editions Bellarmine, Montreal.

HERNÁNDEZ, Alberto. 1989. Sociogeografia de acción de las sociedades religiosas protestantes y paracristianas en cinco ciudades fronterizas. Ponencia presentada en el "V Encuentro Nacional: Estado, Iglesia y Grupos Laicos", en Guadalajara, Jalisco, 611 de noviembre de 1989.

La Atalaya, 1 de enero de 1991, Watch Tower Bible and Tract Society of Nueva York. Grupo Editorial Ultramar, México.

LAMOND Tullis, F. 1987. Mormons in México. The Dynamics of Faith and Culture. Utah State University Press.

Ley de asociaciones religiosas y culto público, del 13 de julio de 1992, en: Diario oficial, 15 de julio de 1992.

MACÍN, Raúl. 1983. Lutero, presencia religiosa y política en México, primera edición. Ediciones Nuevomar, México.

MANWARING, David R. 1962. Render Unto Caesar, the Flag-Salute Controversy. Colegios Hobart y William Smith.

MASFERRER, Elio. 1991. "Nuevos movimientos y tendencia religiosas en América Latina", en: Religiones latinoamericanas, enero-junio 1991, México.

MOLINA Hernández, José Luis, Martha E. Gutiérrez y Carlos Vásquez Malo. 1988. "Sociedades religiosas cristianas no católicas. Estudio descriptivo, Mexicali, 1988", tesis de licenciatura. Escuela de Ciencias Sociales y Políticas de la Universidad Autónoma de Baja California.

MOLINA Hernández, José Luis. 1993. "Desarrollo urbano y campo religioso, Tres perspectivas de análisis". Tesis de maestría. Facultad de Arquitectura-Instituto de Investigaciones Sociales, UABC, Mexicali.

NEILL, Alexander S. 1975. Corazones, no sólo cabezas en la escuela. Editores Mexicanos Unidos, México.

PAZ, Octavio. 1975. El laberinto de la soledad. FCE, México.

REYES Esparza, Ramiro. 1988. "La formación de los maestros en la década de los cuarenta", en: Pedagogía, núm. 16, octubre-diciembre de 1988, UPN, México. 
RODRÍGUEZ Brandao, Carlos. s/f. "Creencia e identidad. Campo religioso y cambio cultural", en: Estudios sobre las culturas contemporáneas, vol. III, núm. 7, Colima.

SELVA Pallares, Dr. Julio Édgar. 1992. "Los testigos de Jehová y la transfusión sanguínea", en: Zeta, del 8 al 14 de mayo de 1992, sección "B", Tijuana.

SHUTZ, Alfred. 1974. El problema de la realidad social. Amorrortu Editores, Buenos Aires.

VALDERREY Falagán, José. 1988. Los nuevos movimientos religiosos en el contexto mundial y latinoamericano. Ponencia presentada en la "XLII Asamblea General de la Conferencia Episcopal Mexicana", México.

WILLIAMS, Paul J. 1952. What Americans Believe and How They Worship. s.d.

WILSON, Bryan. 1970. Sociología de las sectas religiosas. Ediciones Guadarrama. Madrid.

WOODROW, Alain. 1979. Las nuevas sectas, 1ra. edición, FCB, México.

ZÚÑIGA, Rosa María. 1993a. "Un imaginario alienante: la formación de maestros", en: Cero en conducta, año 8, núms. 33-34, mayo de 1993, México.

- 1993b. "El imaginario normalista", en: Cero en conducta, año 8, núms. 33-34, mayo de 1993, México. 